INPLASY

PROTOCOL

To cite: Li et al. Baduanjin exercise for patients with knee osteoarthritis: a protocol for systematic review and metaanalysis. Inplasy protocol 202090051. doi:

10.37766/inplasy2020.9.0051

Received: 11 September 2020

Published: 12 September 2020

Corresponding author: Jieying Li

lijieying59@163.com

Author Affiliation:

Henan University of Chinese Medicine

Review Stage at time of this submission: Preliminary searches.

Conflicts of interest: None.

\section{Baduanjin exercise for patients with knee osteoarthritis: a protocol for systematic review and meta-analysis}

Li, JY1; Yin, S2; Li, RQ3; Ge, BB4; Su, KQ5; Song, XL6; Zhang, ZH; Chang, Y8; Feng, XD9; $\mathrm{Wu}, \mathrm{N}^{10}$.

Review question / Objective: Can Baduanjin exercise benefit for treating knee osteoarthritis?

Condition being studied: Knee osteoarthritis (KOA) is a common chronic degenerative disorder and the leading cause of pain in the elderly. KOA is characterized by progressive loss of articular cartilage, subchondral bone thickening, bone hypertrophy, and new bone formation. Pain symptoms and physical function disability are the primary clinical symptoms of KOA. In addition, with disease progression, KOA may impair patients'normal quality of life. Baduanjin is a traditional chinese mind-body exercise routine.Some Data came from papers and researches reveal that Baduanjin exercises produce a great effect on improving the pain, stiffness and mobility of KOA patients. while some other reports indicate that there are no significant differences for effects of KOA by practicing Baduanjin. Therefore, This study will summarizes and evaluates the effect of existing clinical trials on the KOA by Baduanjin.

INPLASY registration number: This protocol was registered with the International Platform of Registered Systematic Review and Meta-Analysis Protocols (INPLASY) on 12 September 2020 and was last updated on 22 September 2020 (registration number INPLASY202090051).

\section{INTRODUCTION}

Review question Baduanjin exercise knee osteoarthritis?
Condition being studied: Knee osteoarthritis (KOA) is a common chronic degenerative disorder and the leading cause of pain in the elderly. KOA is characterized by progressive loss of articular cartilage, subchondral bone thickening, bone hypertrophy, and new 
bone formation. Pain symptoms and physical function disability are the primary clinical symptoms of KOA. In addition, with disease progression, KOA may impair patients'normal quality of life. Baduanjin is a traditional chinese mind-body exercise routine.Some Data came from papers and researches reveal that Baduanjin exercises produce a great effect on improving the pain, stiffness and mobility of KOA patients. while some other reports indicate that there are no significant differences for effects of KOA by practicing Baduanjin. Therefore, This study will summarizes and evaluates the effect of existing clinical trials on the KOA by Baduanjin.

\section{METHODS}

Search strategy: The search terms are: ('Osteoarthritides'OR'Osteoarthrosis'OR 'Osteoarthroses' OR'Arthritis, Degenerative'OR'Arthritides, Degenerative' OR'Degenerative Arthritides' OR 'Degenerative Arthritis' OR 'Arthrosis' OR 'Arthroses'OR'Osteoarthrosis Deformans') AND ('Baduanjin' OR'eight section brocades' OR'eight-section Brocade' OR 'eight section brocades' OR 'eight trigrams boxing' OR 'eight-treasured exercises' OR 'eight pieces of brocade'OR 'Eight Brocade Section' Baduanjin exercises'OR 'Baduanjin exercise' OR 'Baduanjin Qigong'). Chinese translations of these terms will be applied to Chinese database. Initially, and to increase the chance of identifying all relevant papers, the search will not be limited to any specific criteria. Articles published in English and Chinese will be considered. The reference list of the identified papers will also be searched. The identified articles will be imported into the EndNote reference management software. This software will help to identify any duplicates. The titles and abstracts will be screened. We will retrieve the full text of the relevant papers for further assessment.

Participant or population: Patients
diagnosed with KOA.

Intervention: Studies were included if Baduanjin exercise was used as the sole intervention.
Comparator: A waiting list, placebo, no intervention, educational classes, healthcare routine, or other behavioral as controls was included.And we excluded studies in which other Exercise Therapy and other traditional Chinese medicine (TCM) therapies (e.g. moxibustion, Chinese Tuina, acupuncture, Chinese herbals) and Western medicine therapy.

Study designs to be included: The type of study was clinical randomized controlled trial (RCT).

Eligibility criteria: Eligibility criteria were detailed using the Participants, Interventions, Controls, Outcomes, and Studies (PICOS) framework.

Information sources: We will perform medical retrieval in the following database: PubMed, EMBASE, Cochrane Library, Web of Science, National Knowledge Infrastructure (CNKI), Wanfang Data Information Site, Chinese BioMedical Database(CBM), Chinese Science and Technique Journals Database (VIP).

Main outcome(s): The Western Ontario and McMaster Universities Osteoarthritis Index (WOMAC) used to assess pain,stiffness and physical functioning.

Additional outcome(s): The Visual Analogue Scale (VAS); Knee Injury and Osteoarthritis Outcome Score (KOOS); the Medical Outcomes Study Short Form-36 (SF-36); six-minute walk test (6-MWT); active range of motion (AROM) ; Berg Balance Scale (BBS); the Isokinetic Strength of the Knee Extensors (ISKE); Lequesne \&Mery Index.

Data management: Two reviewers will independently assess the eligibility of the studies retrieved against the inclusion and exclusion criteria. Then those studies meeting the criteria will be selected for use in the review.The following data will then be extacted from the studies selected for 
inclusion using a data collection form,and recorded in an Excel file:(1) Details of study: first author, year of publication;(2) Study population: age, sex, sample size;(3) Intervention characteristics: type, frequency and duration;(4) Outcome measures: Clinical effective rate, WOMAC, VAS, The KOOS, adverse effects and so on.

Quality assessment / Risk of bias analysis: Two of our researchers (Shuai Yin and Xiaolei Song) will respectively use the bias risk tool (Cochrane Handbook for Systematic Reviews of Interventions 5.3) provided by the Cochrane Collaboration to evaluate the quality of the included literature. Seven domains should be evaluated, including random sequence generation, allocation concealment, blinding of participants and personnel, blinding of outcome assessment, incomplete outcome data, selective reporting of positive and/or negative findings, and other sources of bias. If the 2 researchers differ in determining the bias, the differences are resolved through discussion. If there is still no consensus after discussion, we will seek advice from a third part (Xiaodong Feng and Nan Wu). Only literature with a score greater than 5 will be included. And another two authors (Ruiqing $\mathrm{Li}$ and Kaiqi $\mathrm{Su}$ ) will assess the quality of the evidence using the GRADE framework, covering study limitations, inconsistencies, indirectness, imprecision and publication biases.

Strategy of data synthesis: We will perform the Cochrane's Review Manager software (V.5.3) to analyse statistics. It is used $95 \%$ confidence intervals (Cls) to the mean difference (MD) or standardized mean difference (SMD) to analyze continuous outcomes. About the assess to heterogeneity and to choose the effect model will take $I^{2}$ statistic and $X^{2}$-test adopting. When $\mathrm{I}^{2}$ statistic $>50 \%$ and $\mathrm{P}$ value less than 0.1 that we consider heterogeneity exists in research at the same time a random-effects model was selected. On the contrary, the selection of a fixed-effects model.
Subgroup analysis: Subgroup analysis will be carried out if significant levels of heterogeneity, or any incongruities, are detected within the analysis.

Sensibility analysis: Sensitivity analysis may be performed by removing low quality studies, or trials with a short-term followup.

Language: We will include reports of randomized controlled trials (RCTS) conducted in English or Chinese.

Country(ies) involved: China.

Keywords: Baduanjin exercise, knee osteoarthritis, systematic review, metaanalysis.

Support: This study is supported by Science and Technology Department of Henan Province (182102310311, 192102310162), Special Project of Scientific Research on Traditional Chinese Medicine in Henan Province (grant number

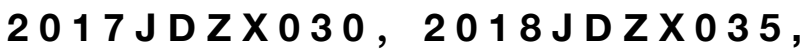
2018JDZX115, 2019JDZX2001). Doctoral Research Fund of Henan University of Chinese Medicine (BSJJ2018-12), Henan Provincial Training Program for Leading Talents in Clinical Discipline of Traditional Chinese Medicine (2100202).

Contributions of each author:

Author 1 - Jieying Li.

Author 2 - Shuai Yin.

Author 3 - Ruiqing Li.

Author 4 - Beibei Ge.

Author 5 - Kaiqi Su.

Author 6 - Xiaolei Song.

Author 7 - Zhenhua Zhang.

Author 8 - Yiniu Chang.

Author 9 - Xiaodong Feng.

Author 10 - Nan Wu. 